public awareness survey highlight low awareness which need to be tackled in order to drive earlier diagnosis. This will form key messaging in our new national awareness campaign called ChildCancerSmart.

\section{G176 PUBLIC AWARENESS OF CHILDHOOD, TEENAGERS AND YOUNG ADULT CANCER SIGNS AND SYMPTOMS IN GREAT BRITAIN}

${ }^{1}$ JF Liu, 'D Shanmugavadivel, ${ }^{2} \mathrm{~A}$ Gamble, ${ }^{2} \mathrm{~A}$ Stewart, ${ }^{1} \mathrm{DA}$ Walker. ${ }^{1}$ Children's Brain Tumour Research Centre, University of Nottingham, Nottingham, UK; ' ${ }^{2}$ Children's Cancer and Leukaemia Group, Leicester, UK

\subsection{6/archdischild-2020-rcpch. 147}

Aims To assess public awareness of the risks and symptoms of cancer in children and young adults under 18 in Great Britain and compare them with the published data where $60-94 \%$ of adults are aware of their cancer risks and symptoms.

Methods A face-to-face, computer-assisted opinion survey was conducted by Ipsos MORI. The population-based sample included 475 males and 525 females over the age of 18 and $26 \%$ have children aged 6-15 in the household. Questions covered perception about cumulative cancer risk ( 1 in 450 by the age 15 and 1 in 180 by 25), confidence in recognising signs and symptoms, recognition and perceived urgency of 42 classical signs and symptoms.

Results Over half (56\%) of the respondents felt the quoted cumulative age-related cancer risks were higher than they expected. Only $32 \%$ felt confident to recognise the signs and symptoms of cancer in the age range. On average, respondents identified 14.9 out of the 42 of the classical signs and symptoms. Top ranked symptoms or signs were: 'lump, swelling in pelvis, testicle or breast' (46\%), 'blood in urine or stool' $44 \%$, 'changes to moles' $43 \%$, 'lump or swelling in the chest wall or armpits' $41 \%$ and 'weight loss' $40 \%$. The least recognised symptoms were early or late puberty (10\%), developmental delay in young children under $2(11 \%)$ and slow growth (13\%), where $8 \%, 2 \%$ and $6 \%$, respectively, felt that there was no need to discuss them with a doctor. Symptoms where more than $50 \%$ of respondents judged them as requiring medical assessment within 48 hours were: seizures/fits, blood in urine or stool, persistent vomiting, persistent fever or night sweats. Abnormal facial movements and persistent abdominal pain were added for assessment within 2 weeks. All but one of the symptoms were selected for assessment by 3 months.

Conclusions We conclude that public awareness of the risks and signs and symptoms of childhood cancer are substantially lower than awareness for adult cancer population in Great Britain. A similar survey will be conducted amongst healthcare professionals to support the development of campaign materials for a public and professional awareness programme.

\section{G177 CISPLATIN-INDUCED OTOTOXICITY IN PAEDIATRIC AND ADOLESCENT OSTEOSARCOMA PATIENTS REMAINS A CHALLENGE}

C Soto, EA Corley, M Michelagnoli. Children and Young People's Cancer Service, UCL Hospitals, London, UK

10.1136/archdischild-2020-rcpch. 148
Background/Objectives Cisplatin-induced ototoxicity is a wellrecognised complication of cancer treatment, resulting in nonreversible, often progressive hearing loss.

Risk factors for cisplatin-induced hearing loss include higher cumulative cisplatin dose, younger patient age, head/neck radiotherapy and concomitant use of aminoglycosides. Osteosarcoma typically affects long-bones in later childhood and adolescence; radiotherapy has a limited role and local protocols prohibit routine use of aminoglycosides. We examined whether cisplatin-induced ototoxicity remained a significant side-effect in this lower risk patient group in a retrospective cohort of patients treated as per the EURAMOS-1 protocol (cumulative cisplatin dose $480 \mathrm{mg} / \mathrm{m}^{2}$ ).

Methods Paediatric and adolescent osteosarcoma patients at a single UK tertiary centre over a five-year period (2013 2017) were included. Clinical information and results of puretone audiograms were extracted from patient records. Common Terminology Criteria for Adverse Events version 5 definitions were used to describe ototoxicity; extended high frequency hearing loss (EHFHL) was defined as range $>$ $8 \mathrm{KHz}$.

Results We identified 72 eligible patients with mean age of 12.9 years (median 16 years, range $2-18$ years) and equal gender distribution. 3 patients progressed/relapsed prior to cycle 4 and a further 3 progressed/relapsed prior to their end of treatment assessment. Baseline audiometry was recorded in 59 patients $(82 \%)$.

Of 53 patients who had audiometry before their 4th cycle of cisplatin; EHFHL was seen in 13 (25\%), 6 (11\%) had Grade 1 and 7 (13\%) had Grade 2 hearing loss. 37 patients underwent an end of treatment (EOT) audiogram; 13 (35\%) had some degree of hearing loss and $5(13 \%)$ had grade $\geq 3$. Of the patients with demonstrated hearing loss after three cycles of cisplatin, who had an EOT assessment, 6 demonstrated worsening audiometry.

Conclusion The finding that over half the patients in this lower risk cohort experienced some degree of hearing loss at end of treatment illustrates that ototoxicity remains a significant complication of cisplatin treatment and highlights the importance of rigorous audiometry throughout and following EOT. Since cisplatin has a central role in osteosarcoma prompt recognition of ototoxicity and early institution of support measures is essential and, ultimately, management options to minimise risk of ototoxicity are urgently needed.

\section{G178 LOW-LEVEL LASER THERAPY FOR CHILDREN AND YOUNG PEOPLE UNDERGOING TREATMENT FOR CANCER}

${ }^{1,2} \mathrm{M}$ Redman, ${ }^{3} \mathrm{~K}$ Harris, ${ }^{4} \mathrm{~B}$ Phillips. 'Oncology and Metabolism, University of Sheffield, Sheffield, UK; ${ }^{2}$ Paediatrics, Sheffield Children's NHS Foundation Trust, Sheffield, UK; ${ }^{3}$ Medical Education, University of Liverpool, Liverpool, UK; ${ }^{4}$ Centre for Reviews and Dissemination, University of York, York, UK

\subsection{6/archdischild-2020-rcpch. 149}

Aim To assess the efficacy and tolerance/safety of oral lowlevel laser therapy to prevent or treat oral mucositis in children and young people undergoing chemotherapy.

Introduction Inflammation and ulceration of the oral cavity known as oral mucositis - can affect up to $80 \%$ of children and young people (CYP) undergoing chemotherapy. It can lead to severe oral pain as well as difficulty talking, eating and drinking; it may also lead to treatment cessation. 